(SUPPORTING INFORMATION)

\title{
Chemical Synthesis of Six Novel 17ß-Estradiol and Estrone Dimers and Study of their Formation Catalyzed by Human Cytochrome P450 Isoforms ${ }^{1}$
}

\author{
Aaron Yun Chen, Anthony J. Lee, Xiang-Rong Jiang and Bao Ting Zhu ${ }^{3}$ \\ Department of Pharmacology, Toxicology and Therapeutics, School of Medicine, \\ University of Kansas Medical Center, Kansas City, KS 66160, USA
}

CONTENTS OF THE SUPPORTING INFORMATION

- GC-MS analysis of compounds 1

- $\quad$ GC-MS analysis of compounds 2

- GC-MS analysis of compounds 3

- $\quad$ GC-MS analysis of compounds 4

- $\quad$ GC-MS analysis of compounds 6

- GC-MS analysis of compounds 8 


\section{METHODS}

\section{Equipments and derivatization method}

The gas chromatography-mass spectroscopy (GC-MS) system was composed of Agilent Technology model 6890N GC system, model 5973 mass selective detector and 7683B autosampler. The software used was MSD ChemStation version D.03.00.611.

Column used in this study was HP-5MS 5\% Phenyl Methyl Siloxane, $30 \mathrm{~m} * 250 \mu \mathrm{m} *$ $0.25 \mu \mathrm{m}$ (Agilent).

BSTFA with 1\% TMCS (Supelco) was used as a derivatization reagent for GC-MS analysis. The incubation was performed at $65^{\circ} \mathrm{C}$ for $40 \mathrm{~min}$.

\section{Temperature program used for the GC/MS system}

\begin{tabular}{|c|c|c|c|}
\hline & Heating $\left({ }^{\circ} \mathrm{C} / \mathrm{min}\right)$ & Temperature $\left({ }^{\circ} \mathrm{C}\right)$ & Duration (min) \\
\hline Initial & - & 180 & 0 \\
\hline Ramp 1 & 4 & 260 & 40 \\
\hline Ramp 2 & 5 & 300 & 5 \\
\hline
\end{tabular}

\section{Detector setting}

The scan mode $(\mathrm{m} / \mathrm{z}=50-600)$ was used to test the purity of each synthesized samples. The solvent delay was set to $18 \mathrm{~min}$. 


\section{RESULTS}

The GC-MS spectra of all the six synthesized products (compound $\mathbf{1 - 4 , 6}$ and $\mathbf{8}$ ) were presented below in Fig. 1 -6, respectively. Under the conditions used in the present study, all these six synthesized compounds can be perfectly separated. Note that the retention times of $E_{1}$ and $E_{2}$ was 18.7 and 19.6 min, respectively. The GC-MS peaks and mass spectra of all six synthesized products showed a purity of $>95 \%$. The isomeric impurities (at the $\mathrm{C}-2$ and $\mathrm{C}-4$ positions) contained in these six samples were below $2 \%$. 
TABLE 1. The purity of Compounds 1, 2, 3, 4, 6 and 8 that were synthesized and purified in the present study.

\begin{tabular}{|c|c|}
\hline Compound & Purity \\
\hline 1 & $>95 \%$ \\
\hline 2 & $>95 \%$ \\
\hline 3 & $>95 \%$ \\
\hline 4 & $>95 \%$ \\
\hline 6 & $>95 \%$ \\
\hline 8 & $>95 \%$ \\
\hline
\end{tabular}




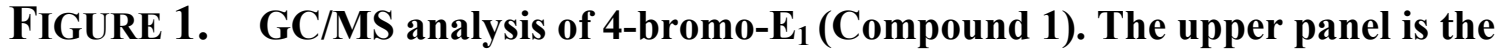
GC chromatograph showing a peak (purity $>95 \%$ ) with a retention time of 25.535 min, and the lower panel is the MS spectrum of its TMS-derivative.
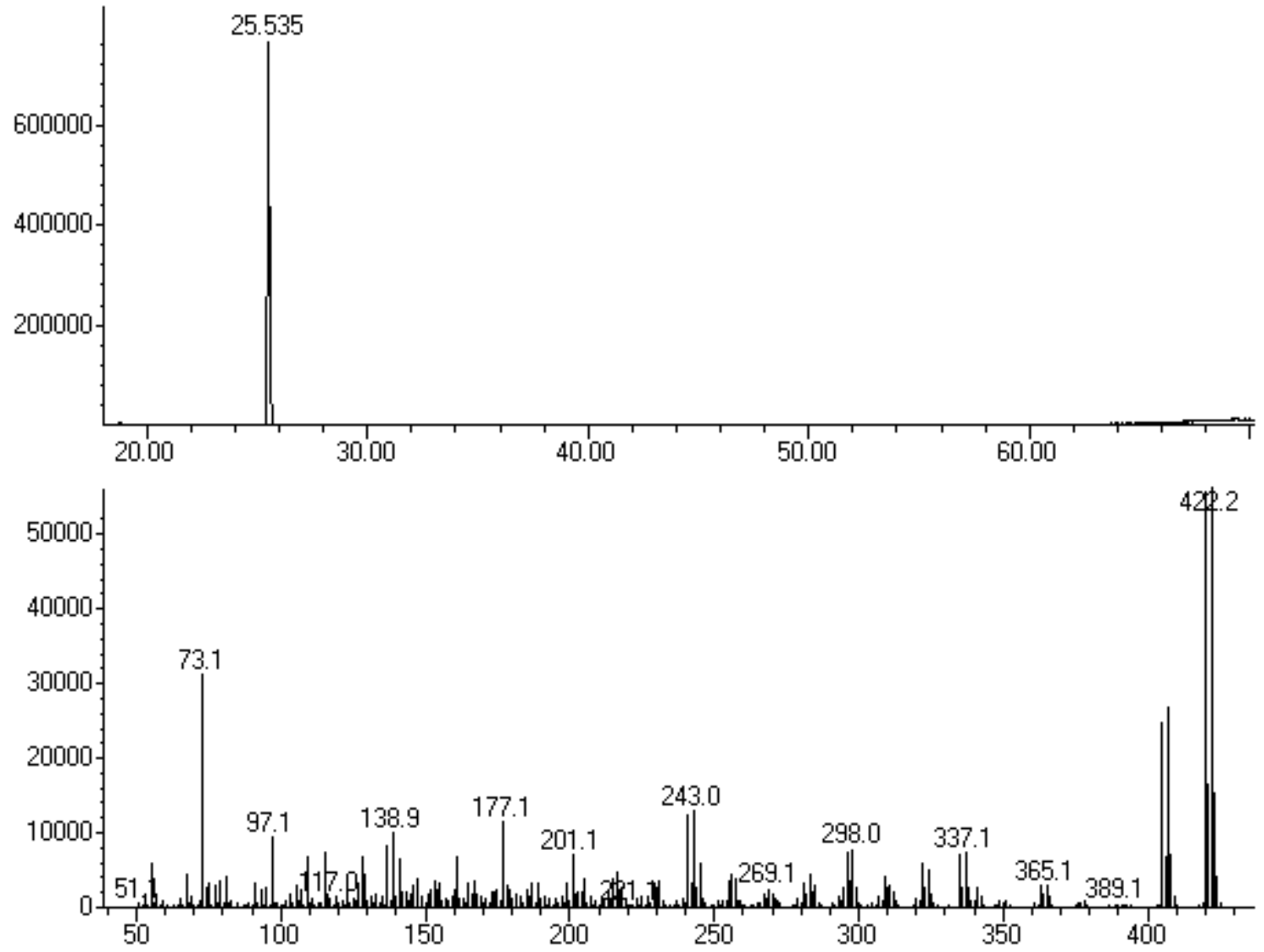
FIGURE 2. GC/MS analysis of 4-bromo- $\mathrm{E}_{2}$ (Compound 2). The upper panel is the GC chromatograph showing a peak (purity $>95 \%$ ) with a retention time of 26.958 min, and the lower panel is the MS spectrum of its TMS-derivative.
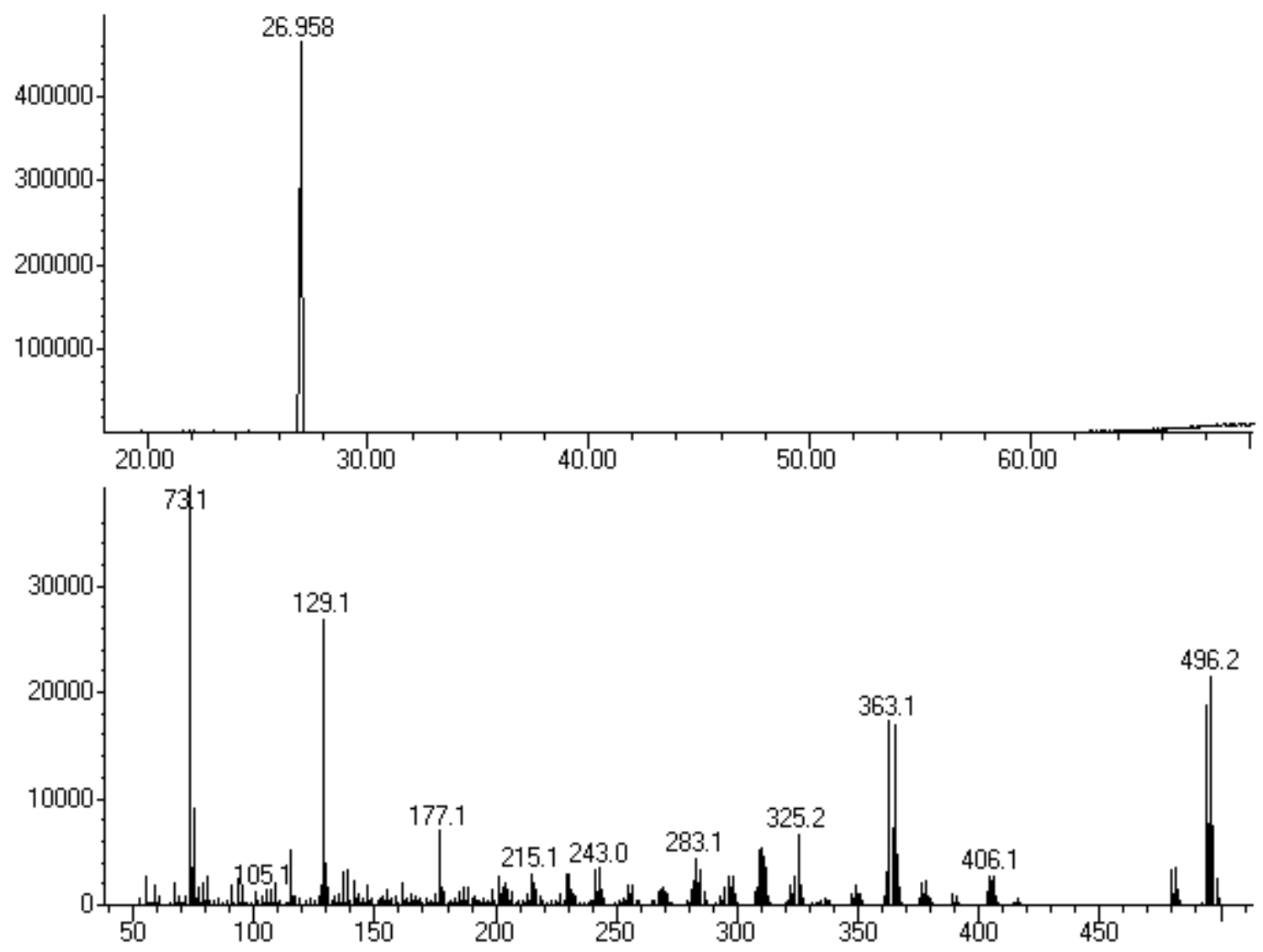
FIGURE 3. GC/MS analysis of 2-bromo- $\mathrm{E}_{1}$ (Compound 3). The upper panel is the GC chromatograph showing a peak (purity $>95 \%$ ) with a retention time of 24.823 min, and the lower panel is the MS spectrum of its TMS-derivative.

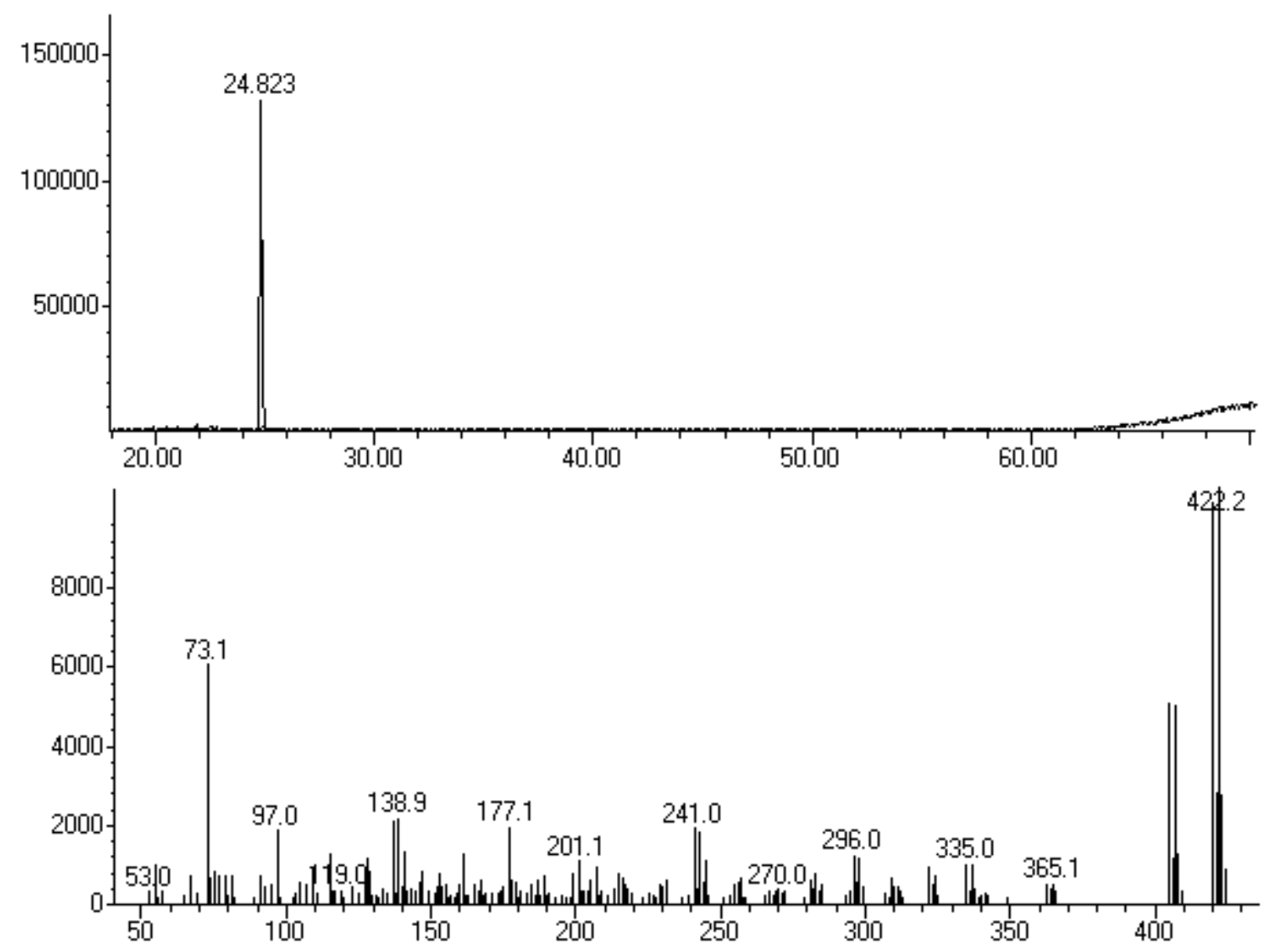




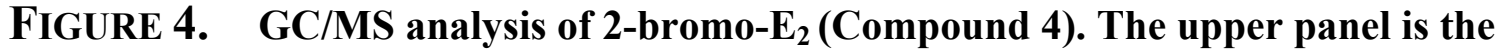
GC chromatograph showing a peak (purity $>95 \%$ ) with a retention time of 26.127 min, and the lower panel is the MS spectrum of its TMS-derivative.
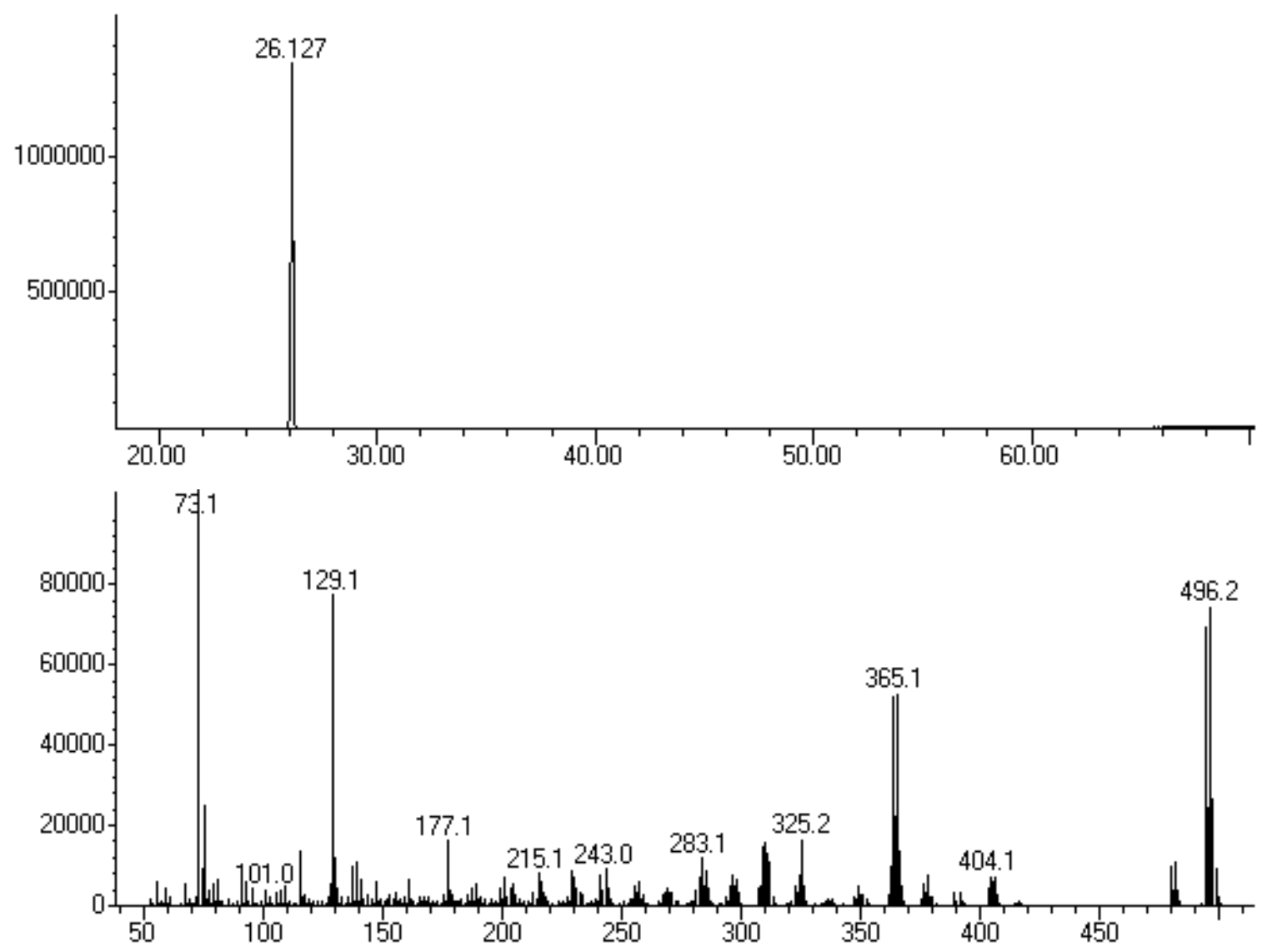


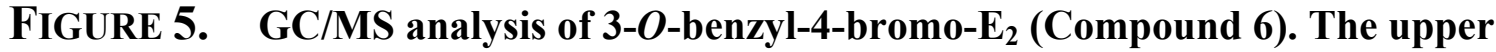
panel is the GC chromatograph showing a peak (purity $>95 \%$ ) with a retention time of $67.793 \mathrm{~min}$, and the lower panel is the MS spectrum of its TMS-derivative.

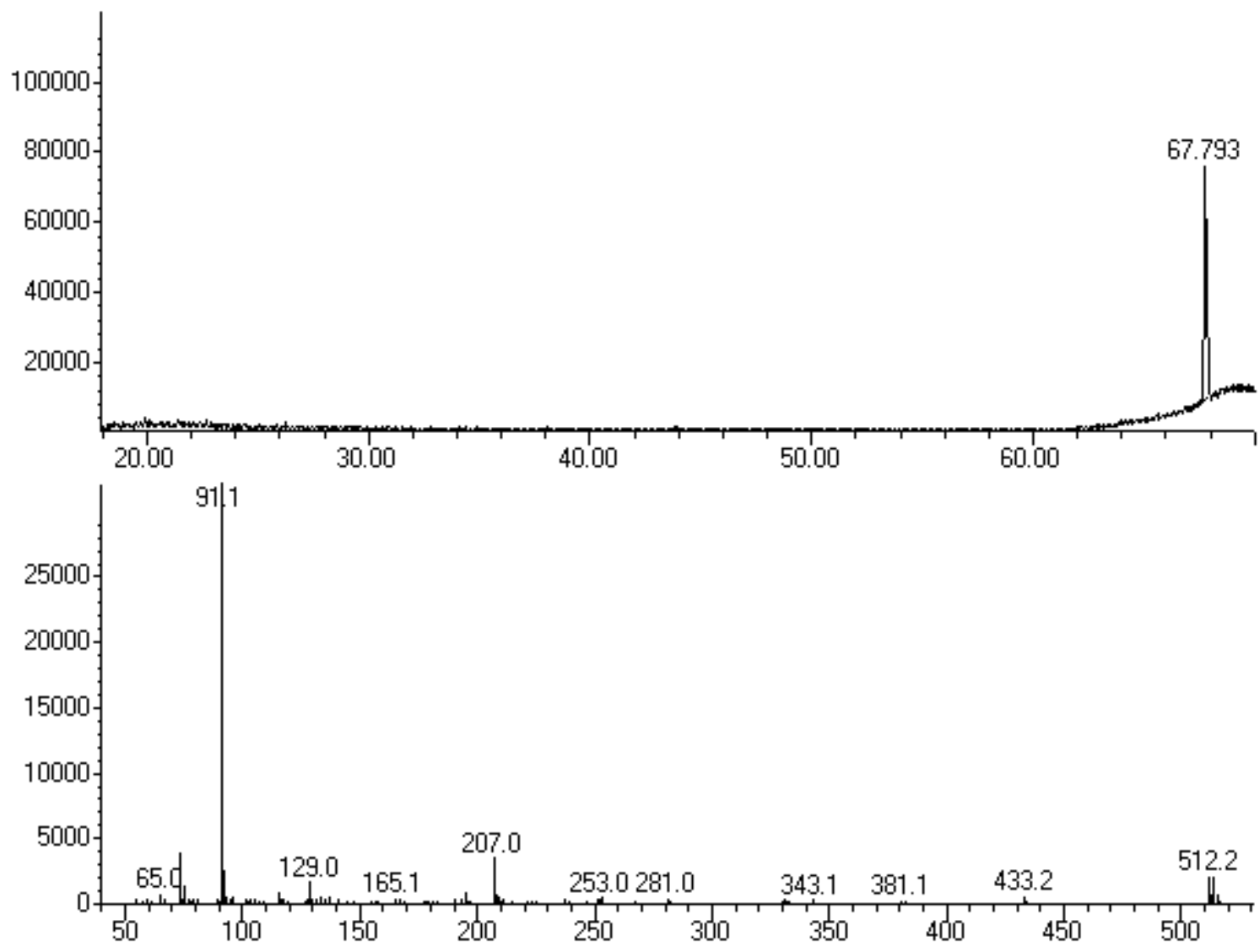




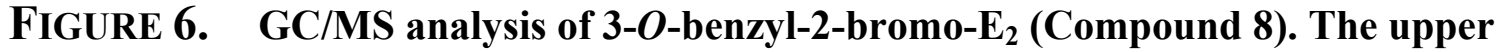
panel is the GC chromatograph showing a peak (purity $>95 \%$ ) with a retention time of $66.269 \mathrm{~min}$, and the lower panel is the MS spectrum of its TMS-derivative.

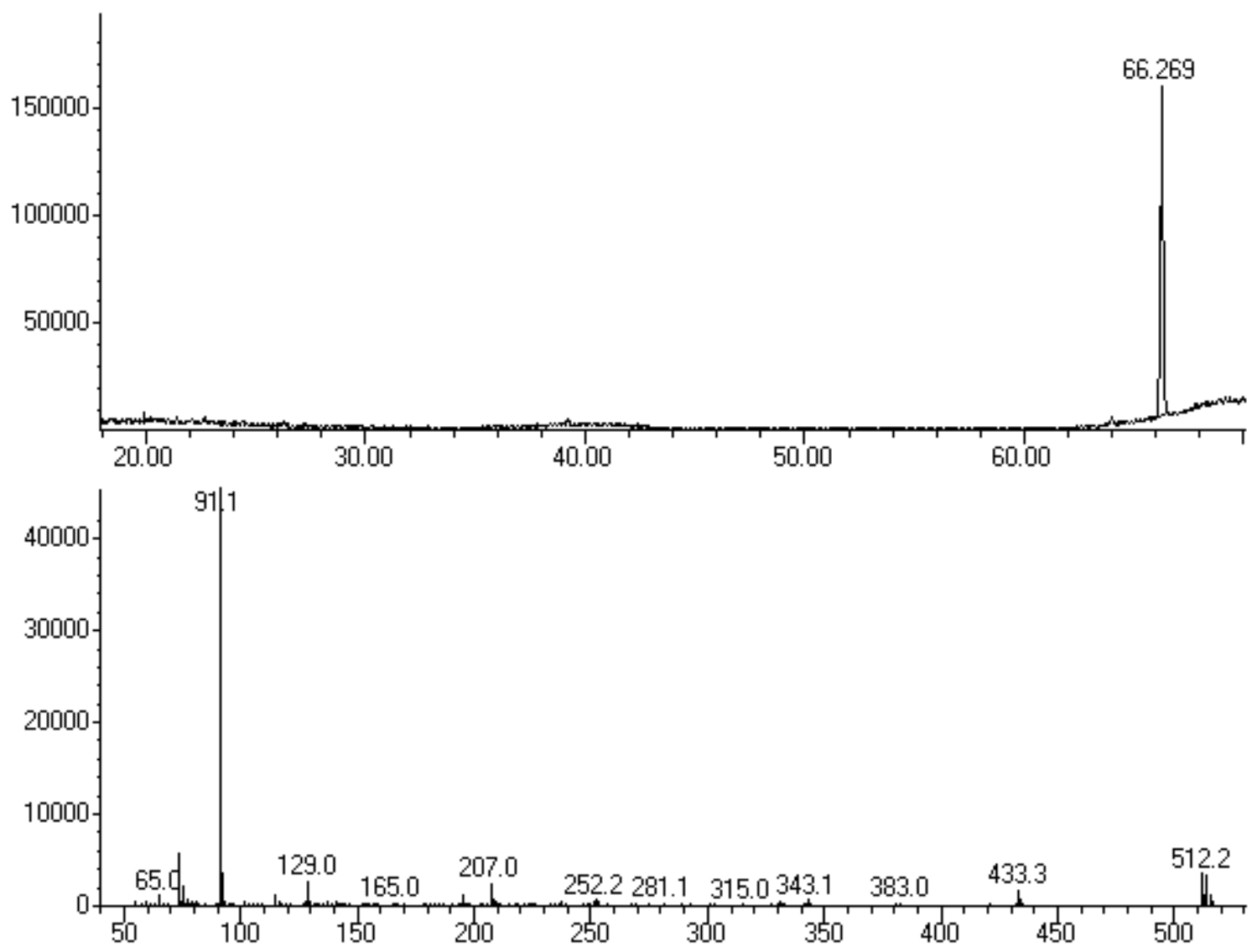

